\title{
EFECTO DE LA DENSIDAD DE POBLACIÓN EN LA CALIDAD DEL GRANO, NIXTAMAL Y TORTILLA DE HÍBRIDOS DE MAÍZ DE ALTA CALIDAD PROTEÍNICA
}

\author{
EFFECT OF PLANT DENSITY ON KERNEL, NIXTAMAL AND TORTILLA QUALITIES \\ OF HIGH QUALITY PROTEIN MAIZE HYBRIDS
}

\author{
Ma. Gricelda Vázquez Carrillo ${ }^{1 \star}$, Hugo Mejía Andrade ${ }^{2}$, Yolanda Salinas Moreno ${ }^{1}$ \\ y David Santiago Ramos ${ }^{1}$
}

${ }^{1}$ Laboratorio de Calidad de Maíz, ${ }^{2}$ Campo Experimental Valle de México, Instituto Nacional de Investigaciones Forestales, Agrícolas y Pecuarias (INIFAP). Km. 13.5 Carr. Los Reyes-Texcoco. 56250, Coatlinchan, Texcoco, Edo. de Méx. Tel. (595) 92 12657, 92 12726, Ext. 106.

${ }^{*}$ Autor para correspondencia (gricelda_vazquez@yahoo.com)

\section{RESUMEN}

Aquí se estudió el efecto de la densidad de población en la calidad comercial y proteínica del grano y las tortillas de tres híbridos de maíz (Zea mays L.), dos de alta calidad proteínica (ACP) desarrollados para los Valles Altos de México ('H-143C' y 'H-149C'), y un testigo no ACP ('Promesa'), cultivados a densidades de 67000 y 80000 plantas ha $^{-1}$. La calidad comercial y la composición química de los híbridos ACP fueron afectadas por la densidad de plantación y su interacción con el genotipo. El manejo del cultivo con 67000 plantas ha $^{-1}$ permitió la producción de maíces ACP con calidad para las industrias de la masa y la tortilla. El híbrido 'H-143C' mostró una calidad semejante en las dos densidades de población estudiadas, mientras que el ' $\mathrm{H}-149 \mathrm{C}$ ' sembrado a 80000 plantas ha ${ }^{-1}$ produjo granos de endospermo suaves, con bajo contenido de proteína $(8.6 \%)$ y triptófano $(0.73 \%)$. Los maíces ACP presentaron una mejor calidad proteínica en comparación con el testigo no ACP. Con los híbridos ACP cultivados a bajas densidades se elaboraron las tortillas recién hechas más blancas y más suaves que el testigo. Los dos híbridos ACP cultivados con 67000 planta ha ${ }^{-1}$ son una excelente alternativa para los industriales de la masa-tortilla, ya que se elaboran tortillas con mejor calidad comercial y proteínica que las del maíz testigo no ACP 'Promesa'.

Palabras clave: Zea mays, densidad de plantación, maíz de alta calidad proteínica, tortilla.

\section{SUMMARY}

The effect of plant density on commercial and protein qualities of kernels and tortillas from three maize hybrids (Zea mays L.) was studied. Hybrids were two quality protein hybrids (QPM) developed for the Highlands of México ('H-143C' and ' $\mathrm{H}-149 \mathrm{C}$ ') and one nonQPM ('Promesa'). All hybrids were grown at 67000 and 80000 plants ha ${ }^{-1}$. Planting density, as well as genotype interaction affected commercial quality and chemical composition of QPM hybrids. Crop management with 67000 plants ha $^{-1}$ allowed production of QPM hybrids with quality for the "masa" (maize dough) and tortilla industries. 'H-143C' hybrid showed similar quality at both planting densities, while ' $\mathrm{H}-149 \mathrm{C}$ ' hybrid produced softer kernels containing low protein $(8.6 \%)$ and tryptophan $(0.73 \%)$ when grown at 80000 plants $\mathrm{ha}^{-1}$. QPM hybrids had better protein quality compared to 'Promesa', the non-QPM hybrid control. Freshly prepared tortillas with QPM hybrids grown at low densities were whiter and softer than the tortillas from the control. The two QPM hybrids grown at density of 67000 plants ha ${ }^{-1}$ are an excellent choice for the "masa" and tortilla industries because their tortillas have improved commercial and protein qualities compared to non-QPM control 'Promesa'.

Index words: Zea mays, quality protein maize, plant density, tortilla.

\section{INTRODUCCIÓN}

Maíz (Zea mays L.) es uno de los cereales de mayor producción a nivel mundial, y es el sostén económico y fuente principal de energía y proteína de millones de personas (FAO, 2013; Melesio et al., 2008). En México es el cultivo más importante, social, económica y nutricionalmente, con aproximadamente 8 millones de hectáreas para su producción (23 millones de toneladas) y constituye $60 \%$ del volumen de granos producidos cada año. Un alto porcentaje de mexicanos tiene al maíz como su principal alimento, con un consumo de $105 \mathrm{~kg}$ de maíz por persona por año) (Polanco y Flores, 2008).

En la región de los Valles Altos de México (altitudes iguales o mayores a $2200 \mathrm{~m}$ ) se siembran 2 millones de hectáreas de maíz, que representan cerca de $25 \%$ de la superficie nacional cultivada con esta especie; las variedades principales corresponden a maíces criollos ( $80 \%$ ), cuya siembra se realiza en bajas densidades de población (40 000 plantas/ ha) y que son susceptibles a enfermedades y al acame de raíz y tallo (Polanco y Flores, 2008).

Los programas de mejoramiento genético en maíz se habían enfocado principalmente al desarrollo de materiales con endospermo normal y alto rendimiento, tolerantes a los factores bióticos y abióticos adversos presentes en la región (Espinosa et al., 2003). No obstante estos maíces son deficientes en los aminoácidos esenciales lisina y triptófano. Con el descubrimiento del gen 02 se revolucionó la producción de maíz ACP, el cual restringe la síntesis de zeínas en el endospermo e incrementa la de otras fracciones proteínicas ricas en estos aminoácidos, lo que duplica su contenido en el grano y el valor biológico de la proteína hasta en $80 \%$ (Gupta et al., 2009). 
Pero los investigadores del Instituto Nacional de Investigaciones Forestales, Agrícolas y Pecuarias (INIFAP) también han desarrollado maíces ACP con las características necesarias para obtener una buena producción y buena calidad para la producción de tortillas de mejor calidad nutricional que las que normalmente se consumen (Vázquez et al., 2012). Con los maíces ACP se busca reducir la desnutrición que padece más de $50 \%$ de la población indígena rural y urbana de bajos ingresos en México (Mazón et al., 2012).

Uno de los factores que afectan el crecimiento y rendimiento del maíz es la densidad de siembra. Para maíz se sugieren densidades de siembra entre 30000 y 90000 plantas $\mathrm{ha}^{-1}$, en función de la región, genotipo, nivel de fertilización y el tipo de riego (Sangoi, 2000). Para el sistema de producción de maíz ACP, algunos autores han obtenido una mayor cantidad de biomasa y mayor rendimiento en grano con densidades entre 73000 y 80000 plantas ha ${ }^{-1}$, aunque el mayor contenido de proteína, lisina y triptófano se ha alcanzado con bajas densidades de población (Vázquez et al., 2005; Mendoza et al., 2006).

El objetivo de esta investigación fue determinar el efecto de la densidad de población en la calidad comercial y proteínica del grano y de las tortillas de híbridos de maíz de alta calidad proteínica ('H-143C' y 'H-149C') desarrollados para los Valles Altos de México.

\section{MATERIALES Y MÉTODOS}

El experimento se condujo bajo condiciones de riego en el ciclo Primavera Verano-2009, en el Campo Experimental Santa Lucía ubicado en el municipio de Texcoco, Estado de México ( $19^{\circ} 29^{\prime} \mathrm{LN}, 98^{\circ} 51^{\prime} \mathrm{LO}$ ), a una altura de 2240 msnm, con una fertilización de $120 \mathrm{~N}-60 \mathrm{P}-00 \mathrm{~K}$. En el sitio predominan los suelos cambrisoles de textura fina y media. La siembra se hizo el 20 de mayo, en dos densidades de población: 67000 (densidad baja, DB) y 80000 (densidad alta, DA) plantas ha ${ }^{-1}$. Los dos híbridos de maíz ACP evaluados fueron 'H-143C' y 'H-149C', ambos de cruzas trilineales y de maduración tardía, en las que se combinaron líneas convertidas a ACP del programa de Valles Altos del INIFAP de alta aptitud combinatoria general (ACG) y líneas ACP proporcionadas por el CIMMYT (Vázquez et al., 2012); se incluyó como testigo comercial el híbrido 'Promesa'.

\section{Análisis físicos y químicos}

Se realizaron en el Laboratorio de Calidad de Maíz del INIFAP. Las variables físicas evaluadas fueron: peso hectolítrico (PH), por el método 84-10 de la AACC (2000); peso de 100 granos (PCG); índice de flotación (IF), como medida indirecta de la dureza del grano; y porcentaje de pedicelo, pericarpio, germen y endospermo, de acuerdo con las metodologías descritas por Salinas y Vázquez (2006). El color en grano se midió en el colorímetro Hunter-Lab MiniScan XE Plus Modelo 45/0-L® (Reston, VA, USA) de acuerdo con la metodología descrita por Salinas y Vázquez (2006). Las variables químicas medidas fueron: contenido de proteína en grano y tortilla, por el método 46-11 (AACC, 2000); contenido de lisina y triptófano en grano y tortillas, con las metodología de Galicia et al. (2009); contenido de aceite en grano entero y en tortilla, por el método 7.044 (AOAC, 1990); y los contenidos de almidón (Herrera y Huber, 1989) y azúcares solubles (Scholes et al., 1994).

\section{Calidad de nixtamal y tortillas}

La nixtamalización, elaboración de tortillas, evaluación de pérdida de sólidos en nejayote (agua de cocción), y medición de la cantidad de pericarpio retenido en el nixtamal se realizaron con las metodologías descritas por Salinas y Vázquez (2006). El tiempo de cocción para cada muestra se asignó de acuerdo con su índice de flotación (IF), como sigue: granos con IF entre 0 y $12 \%$ recibieron 45 min de cocción; con IF entre 13 y $37 \%, 40 \mathrm{~min}$; con IF de 38 a $62 \%, 35 \mathrm{~min}$; y con IF de 63 a $87 \%, 30 \mathrm{~min}$ (Vázquez et al., 2012). En nixtamal, masa y tortillas se midió la humedad por el método 44-10 del AACC (2000). Se cuantificó el rendimiento de tortilla, con base en la cantidad de tortilla obtenida por kilogramo de maíz procesado. En tortillas frías, después de 2 y 24 h de elaboradas, se evaluó la fuerza de punción requerida para romperlas con el texturómetro Brookfield ${ }^{\circledR}$ modelo CT3 (Middleboro, Massachusetts, USA) (Arámbula et al., 2004). El color en tortillas se midió con el colorímetro Hunter Lab® antes descrito.

\section{Análisis estadístico}

Los resultados se analizaron con un diseño completamente aleatorizado con un arreglo factorial para cuantificar el efecto de densidad de población, híbrido y la interacción entre ambos factores. La comparación de medias se hizo con la prueba de Tukey, y también se hizo un análisis de correlación simple entre peso hectolítrico e índice de flotación, entre humedad del nixtamal y humedad de la masa, entre porcentaje de pericarpio retenido y contenido de sólidos en el nejayote, y entre humedad de la tortilla y la fuerza de ruptura. Todos los análisis se hicieron con el paquete estadístico SAS para Windows, versión 9.0.

\section{RESULTADOS Y DISCUSIÓN}

Se observaron diferencias significativas $(\mathrm{P} \leq 0.01)$ entre híbridos en todas las variables evaluadas. La densidad de población (DP) y la interacción DP x híbrido mostraron efectos significativos $(\mathrm{P} \leq 0.05)$ sólo en algunas variables químicas y en características de nixtamalización y calidad de tortillas. 


\section{Características físicas del grano}

La interacción DP $\mathrm{x}$ híbrido fue significativa en el peso hectolítrico $(\mathrm{PH})$, índice de flotación (IF) y color del grano. Los híbridos de maíz ACP sembrados a densidad baja mostraron mayor $\mathrm{PH}$ que el híbrido 'Promesa' usado como testigo. A densidad alta, el híbrido ' $\mathrm{H}-143 \mathrm{C}$ ' tuvo valores de $\mathrm{PH}$ e índice de flotación más altos que el testigo 'Promesa' (Cuadro 1). El híbrido 'H-143C' presentó características semejantes de grano en ambas densidades ( $\mathrm{PH}$ alto, grano pequeño y dureza intermedia). Por su parte, el híbrido ' $\mathrm{H}$ $149 \mathrm{C}$ ' registró mayores valores de $\mathrm{PH}$, granos más grandes y más duros a densidad baja que en densidad alta, pues en ésta redujo su tamaño de grano (peso de 100 granos, PCG) y aumentó su suavidad.

El comportamiento observado en el 'H-149C' indica que este híbrido es más sensible a la competición entre plantas por luz y nutrientes, ya que según Sangoi (2000) y Carrera y Cervantes (2006), a mayores densidades se limita la formación de células y de gránulos de almidón, y por tanto se reducen la capacidad de crecimiento del endospermo y el tamaño del grano, y aumenta la suavidad. En contraparte, el híbrido 'H-143C' aumentó su tamaño de grano (PCG) y dureza (IF), y el testigo sólo su tamaño de grano, cuando fueron cultivados a densidades altas (Cuadro 1), lo que de acuerdo con Sangoi (2000) demuestra una alta tolerancia a la competición entre plantas y al estrés ambiental.

El peso hectolítrico correlacionó negativamente con el índice de flotación $(\mathrm{r}=-0.73 ; \mathrm{P} \leq 0.01)$, en concordancia con lo antes informado por Vázquez et al. (2012). En ambas densidades el testigo 'Promesa' presentó los granos con el menor IF, es decir que fueron más duros que los granos de los híbridos ACP, ya que en éstos predominaron los granos de dureza intermedia (IF = 38 a $62 \%$ ). De acuerdo con Salinas et al. (2010), el maíz blanco destinado a la elaboración de tortillas mediante el método tradicional, debe tener un
PH mínimo de $74 \mathrm{~kg} \mathrm{hL}^{-1}$, valores de $\mathrm{IF} \leq 50$ y $\mathrm{PCG} \leq 33 \mathrm{~g}$, características que cumplen los híbridos ACP sembrados a densidad baja y el híbrido 'H-143C' y el testigo 'Promesa' cultivados a densidad alta, por lo que son adecuados para estas industrias. La suavidad del grano del híbrido 'H-149C' sembrado a 80000 plantas ha-1 limita su procesamiento por las industrias de la masa y la tortilla (IMT), pero su nixtamalización a nivel doméstico es ventajosa, pues Vázquez et al. (2012) indicaron que este tipo de maíces requieren menos tiempo para la nixtamalización y por tanto menos combustible, además de que el moldeado manual de la masa resulta más fácil.

En los componentes estructurales del grano, solo el contenido de germen fue afectado significativamente $(\mathrm{P} \leq 0.01)$ por la interacción DP $\mathrm{x}$ híbrido. Los híbridos 'H-143' y 'H149C' presentaron un mayor contenido de germen cuando fueron cultivados a 67000 plantas ha- ${ }^{-1}$, mientras que a la misma densidad el híbrido 'Promesa' presentó el menor contenido de esta estructura (Cuadro 1). La característica más relevante fue el mayor contenido de pericarpio de los híbridos ACP respecto al testigo, lo cual según Palafox et al. (2008) pudiera representar una barrera más efectiva ante el ataque de insectos de almacén, y además según Martínez et al. (2001) aportaría una mayor cantidad de gomas naturales a la masa con lo que mejoraría la textura de las tortillas.

\section{Calidad de nixtamal y tortillas}

La interacción DP $\mathrm{x}$ híbrido influyó en la pérdida de sólidos en el agua de cocción (nejayote), el pericarpio retenido por el nixtamal y el rendimiento de tortilla fría. La humedad del nixtamal fluctuó entre 42.6 a $50.2 \%$, y la del híbrido 'H-149C' fue la mayor en ambas densidades, mientras que la del ' $\mathrm{H}-143 \mathrm{C}$ ' y el testigo fueron similares entre sí (Cuadro 2). El alto contenido de humedad en el nixtamal del 'H-149C' cultivado a densidad alta se atribuye principalmente a la suavidad de su grano, lo que implica

Cuadro 1. Comparación de medias de las características físicas y componentes estructurales del grano de dos híbridos de alta calidad proteínica (ACP) y un testigo no ACP ('Promesa') producidos en Santa Lucía, Coatlinchán, Estado de México, PV-2009, en dos densidades de población.

\begin{tabular}{|c|c|c|c|c|c|c|c|c|c|}
\hline \multirow{2}{*}{ Densidad (plantas ha-1) } & \multirow{2}{*}{ Híbrido } & \multirow{2}{*}{$\begin{array}{c}\mathrm{PH}^{\dagger} \\
\left(\mathrm{kg} \mathrm{hL}^{-1}\right)\end{array}$} & \multirow{2}{*}{$\mathrm{PCG}^{\dagger \dagger}(\mathrm{g})$} & \multirow{2}{*}{$\operatorname{IF}^{9}(\%)$} & \multirow{2}{*}{$\begin{array}{c}\text { Dureza } \\
\text { grano }\end{array}$} & Pedicelo & $\mathrm{PER}^{\phi}$ & Germen & $\mathrm{END}^{\phi \phi}$ \\
\hline & & & & & & \multicolumn{4}{|c|}{$(\%)$} \\
\hline \multirow{2}{*}{67000} & 'H-143C' & $80.0 \mathrm{a}$ & $23.3 \mathrm{e}$ & $48 \mathrm{~b}$ & $I^{\xi \xi}$ & $1.5 \mathrm{~b}$ & $6.7 \mathrm{a}$ & $10.3 \mathrm{c}$ & $81.5 \mathrm{a}$ \\
\hline & 'Promesa' & $77.4 \mathrm{~b}$ & $31.5 \mathrm{~b}$ & $35 \mathrm{~d}$ & $\mathrm{D}^{\xi}$ & $1.6 \mathrm{ab}$ & $5.6 \mathrm{~b}$ & $11.2 \mathrm{~b}$ & $81.6 \mathrm{a}$ \\
\hline 80000 & 'H-143C' & $79.2 \mathrm{ab}$ & $26.1 \mathrm{~d}$ & $41 \mathrm{bcd}$ & $I^{\xi \xi}$ & $1.7 \mathrm{ab}$ & $6.6 \mathrm{a}$ & $10.2 \mathrm{c}$ & $81.5 \mathrm{a}$ \\
\hline
\end{tabular}

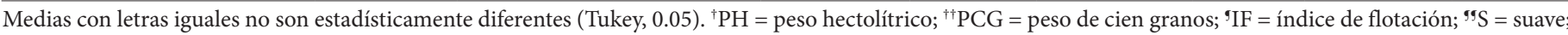
${ }^{\xi} \mathrm{D}=$ duro; ${ }^{\xi \xi} \mathrm{I}=$ intermedio; ${ }^{\phi} \mathrm{PER}=$ pericarpio; ${ }^{\phi} \mathrm{END}=$ endospermo. 
un mayor contenido de endospermo harinoso. La fracción harinosa del endospermo posee mayor cantidad de amilopectina que la fracción vítrea (Dombrink y Knutson, 1997), y la amilopectina absorbe más rápido el agua que la amilosa (Ansari et al., 2010).

En una investigación previa, Vázquez et al. (2012), encontraron que el nixtamal del híbrido 'H-149C', sembrado en Santa Lucía, registró la misma humedad que la registrada aquí (50.2\%), lo que se relacionó con un mayor rendimiento de masa y de tortillas. Las industrias de la masa y la tortilla prefieren maíces cuyo nixtamal alcance humedades entre 42 y 48 \% (Zepeda et al., 2007), por lo que sólo el híbrido 'H-143C' y el testigo 'Promesa' sembrados en las dos densidades cumplen este requisito. La humedad de la masa es un factor importante, cuyo valor óptimo para elaborar tortillas de calidad y con buena vida de anaquel fluctúa entre 50 y 58 \% (Antuna et al., 2008).

En este trabajo el contenido de humedad en la masa estuvo dentro del intervalo mencionado, donde el híbrido ' $\mathrm{H}$ 149C' sobresale con $58.2 \%$ cuando fue cultivado a densidad alta, y correlacionó con la humedad del nixtamal $(\mathrm{r}=0.82$; $\mathrm{P} \leq 0.01)$. Valores semejantes (57.2 a $59.9 \%)$ fueron informados por Serna et al. (2008) para masa de maíces ACP. Los maíces ACP tuvieron una menor pérdida de sólidos en ambas densidades, con respecto al testigo (Cuadro 2), así como un alto porcentaje de pericarpio retenido; ambos resultados se atribuyen al mayor contenido de pericarpio en el grano, por lo que resultan menos susceptible a la hidrólisis por el hidróxido de calcio durante la nixtamalización, lo que reduce así la pérdida de sólidos.

La correlación entre el porcentaje de pericarpio retenido y la pérdida de sólidos fue de $-0.88(\mathrm{P} \leq 0.01)$. Todos los materiales presentaron pérdidas de sólidos menores al valor de $5.0 \%$ demandado en la norma NMX-034 (2002) para maíces destinados a la producción industrial de torti- llas. Las industrias de la masa y la tortilla (IMT) prefieren los maíces cuyo nixtamal retenga más de $30 \%$ de pericarpio (Salinas et al., 2010), por lo que el testigo sembrado a densidades bajas no cumple este requisito. Los mismos autores afirman que para obtener un rendimiento de $1.5 \mathrm{~kg}$ de tortilla por kg de maíz, como lo demandan las IMT, se requiere un contenido de humedad en las tortillas mínimo de $45.0 \%$; en este estudio el mayor rendimiento de tortilla se obtuvo con el híbrido 'H-149C' cultivado en las dos densidades, aun cuando la humedad de sus tortillas fue menor de $45 \%$, lo cual está acorde con lo informado por Vázquez et al. (2012), quienes obtuvieron un rendimiento de $1.59 \mathrm{~kg}$ de tortilla por kg de maíz.

El híbrido ' $\mathrm{H}-143 \mathrm{C}$ ' tuvo los rendimientos de tortilla más bajos en ambas densidades. Una mayor humedad en tortilla se correlacionó positivamente con un mayor rendimiento de éstas $(r=0.96 ; \mathrm{P}<0.01)$. El color del grano guarda una estrecha relación con la calidad de la tortilla en términos de la aceptación por parte del consumidor, que en general son mejor aceptadas las tortillas de color blanco o cremoso brillante (Salinas et al., 2007). En ambas densidades la luminosidad del grano de los híbridos ACP fue mayor que la del testigo 'Promesa', es decir los granos de los ACP fueron más blancos (Figura 1).

La luminosidad de las tortillas recién elaboradas ( $2 \mathrm{~h}$ ) fue mayor en los materiales sembrados a una densidad de 67 000 plantas ha $^{-1}$. Posterior al periodo de almacenamiento refrigerado la luminosidad de las tortillas disminuyó ligeramente, fenómeno que de acuerdo con Salinas et al. (2007) puede ser causado por una mayor concentración de compuestos fenólicos en el pericarpio y endospermo de esos maíces. Este comportamiento solo se observó en las tortillas del híbrido 'H-143C' cultivado en densidad alta, cuya luminosidad aumentó ligeramente (Figura 1). Otro factor importante para la aceptación de la tortilla por los consumidores es la textura, que entre más blanda y suave el

Cuadro 2. Comparación de medias de variables de calidad nixtamalero-tortillera de dos híbridos de alta calidad proteínica (ACP) y un testigo no ACP ('Promesa') producidos en Santa Lucía, Coatlinchán, Edo. de Méx., PV-2009, en dos densidades de población.

\begin{tabular}{|c|c|c|c|c|c|c|}
\hline \multirow{2}{*}{$\begin{array}{l}\text { Densidad } \\
\left(\text { plantas ha }^{-1}\right)\end{array}$} & \multirow{2}{*}{ Híbrido } & \multicolumn{2}{|c|}{ Humedad (\%) } & Sólidos & $\mathrm{PR}^{\dagger}$ & \multirow{2}{*}{$\begin{array}{c}\mathrm{RT}^{\dagger \dagger} \\
\text { (kg tortilla/kg maíz) }\end{array}$} \\
\hline & & Nixtamal & Masa & \multicolumn{2}{|c|}{$(\%)$} & \\
\hline \multirow{3}{*}{67000} & 'H-143C' & $42.6 \mathrm{c}$ & $55.3 \mathrm{bc}$ & $2.5 \mathrm{c}$ & $40.5 \mathrm{a}$ & $1.45 \mathrm{bc}$ \\
\hline & 'H-149C' & $49.3 \mathrm{ab}$ & $57.8 \mathrm{a}$ & $2.9 \mathrm{~b}$ & $41.1 \mathrm{a}$ & $1.53 \mathrm{ab}$ \\
\hline & 'Promesa' & $45.9 \mathrm{bc}$ & $56.9 \mathrm{a}$ & $3.4 \mathrm{a}$ & $28.7 \mathrm{c}$ & $1.49 \mathrm{abc}$ \\
\hline \multirow{3}{*}{80000} & 'H-143C' & $43.6 \mathrm{c}$ & $56.8 \mathrm{ab}$ & $2.8 \mathrm{~b}$ & $34.9 \mathrm{~b}$ & $1.30 \mathrm{~d}$ \\
\hline & 'H-149C' & $50.2 \mathrm{a}$ & $58.2 \mathrm{a}$ & $2.4 \mathrm{c}$ & $41.0 \mathrm{a}$ & $1.60 \mathrm{a}$ \\
\hline & 'Promesa' & $43.8 \mathrm{c}$ & $55.0 \mathrm{c}$ & $3.4 \mathrm{a}$ & $31.9 \mathrm{bc}$ & $1.40 \mathrm{~cd}$ \\
\hline
\end{tabular}

Medias con letras iguales no son estadísticamente diferentes (Tukey, 0.05$) .{ }^{\dagger} \mathrm{PR}=$ pericarpio retenido; $\mathrm{RT}^{\dagger \dagger}=$ rendimiento de tortilla. 
trabajo necesario para su masticación es menor, lo cual es una característica de buena calidad (Antuna et al., 2008). Las tortillas recién hechas más suaves, es decir con la menor fuerza de ruptura, se obtuvieron con el híbrido ' $\mathrm{H}-143 \mathrm{C}$ ' producido en ambas densidades (Figura 2). Las tortillas recién hechas con el híbrido 'Promesa', en ambas densidades, requirieron la mayor fuerza de ruptura. La fuerza requerida para romper las tortillas se correlacionó en forma negativa con su contenido de humedad $(\mathrm{r}=-0.74 ; \mathrm{P} \leq 0.01)$.

Posterior al almacenamiento $(24 \mathrm{~h})$ las tortillas perdieron suavidad, debido principalmente a la retrogradación del almidón, pese a que el contenido de humedad de las tortillas en todos los materiales aumentó entre 1 y $2 \%$ (Figura 2). Este aumento en el contenido de humedad se debe a la condensación del vapor agua que liberan las tortillas dentro de la bolsa de polietileno al pasarlas de la temperatura ambiente a la temperatura de refrigeración durante el almacenamiento.

\section{Calidad proteínica y otros componentes.}

La interacción DP x híbrido influyó en los componentes del grano. El contenido de proteína del grano del híbrido ' $\mathrm{H}-143 \mathrm{C}$ ' no mostró diferencia estadística $(\mathrm{P} \leq 0.01)$ entre densidades de siembra (Cuadro 3 ), lo que indica que la pro- teína en este híbrido depende en mayor medida del genotipo, como también lo indicaron Vázquez et al. (2005); en cambio, el híbrido 'H-149C' tuvo un contenido de proteína más elevado cuando se cultivó en densidad baja (10.4\%) que a densidad alta (8.6\%). Por su parte, el testigo 'Promesa' presentó mayor contenido de proteína a densidad alta que a densidad baja, comportamiento similar al reportado por Luca y Tabara (2010) en otros híbridos no ACP cultivados a densidad alta.

La transformación a tortillas redujo el porcentaje de proteína, y el híbrido 'H-149C' sembrado a densidad baja registró la mayor pérdida (1.3\%). Las pérdidas observadas en el contenido de proteína en las tortillas de éstos híbridos fue inferior al informado previamente por Vázquez et al. (2012). Las tortillas elaboradas con el testigo tuvieron la mayor cantidad de proteína (Cuadro 3). El contenido de lisina y triptófano fue mayor en el grano y las tortillas elaboradas a partir de los híbridos ACP (Cuadro 3), con respecto al testigo 'Promesa', resultados que concuerdan con lo reportado por Sierra et al. (2010) quienes contrastaron la calidad de maíces ACP con maíces no ACP o "de endospermo normal".

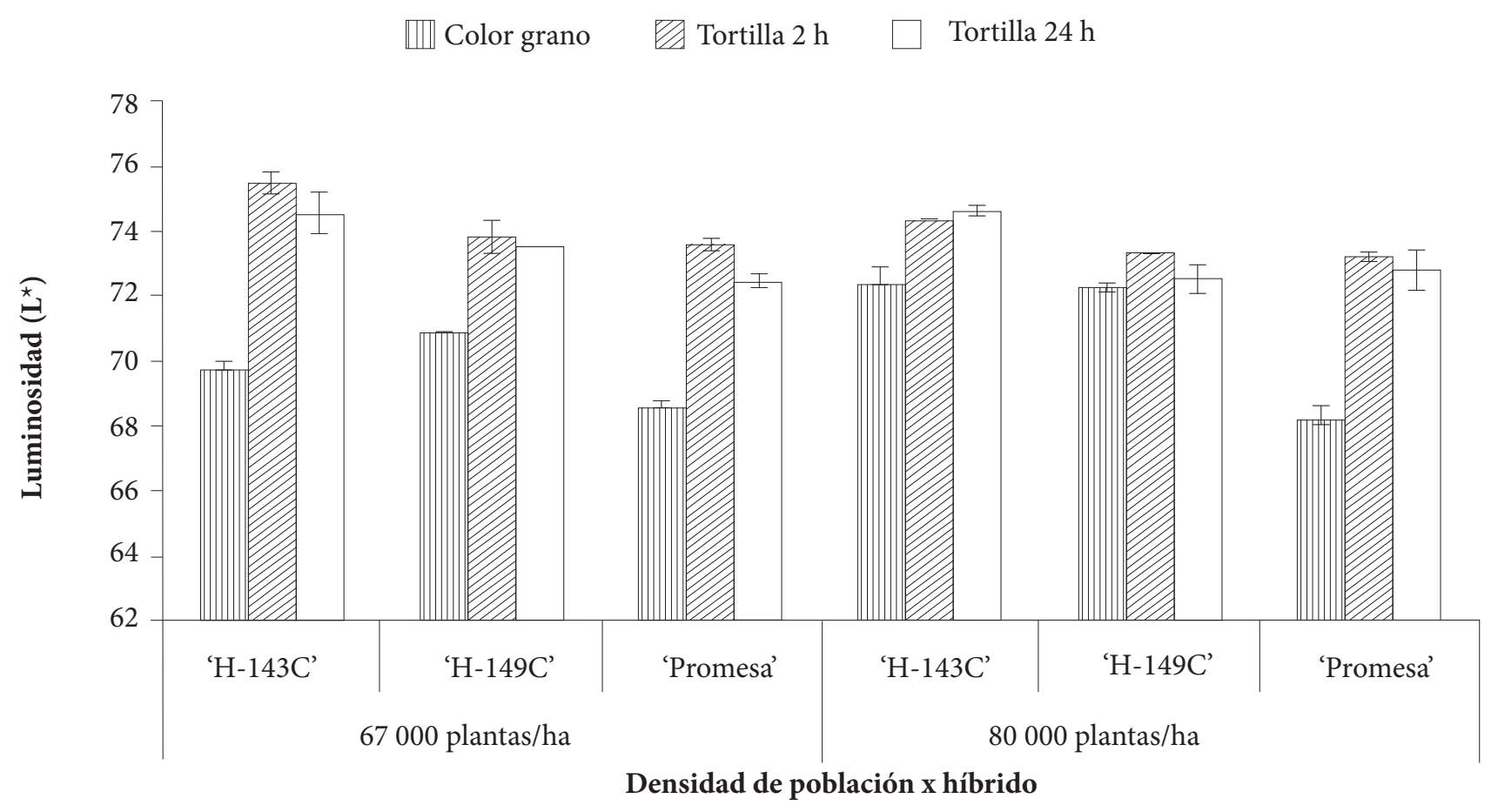

Figura 1. Color en grano y tortillas de dos híbridos de alta calidad proteínica (ACP) y un testigo no ACP ('Promesa') producidos en Santa Lucía, Coatlinchán, Estado de México, PV-2009, en dos densidades de población. 
El contenido de lisina en el grano (expresado en $\mathrm{g}$ de lisina/100 g de proteína) fue superior cuando provino de plantas cultivadas a densidad baja, similar a lo reportado por Mendoza et al. (2007) para híbridos ACP cultivados en distintas densidades. El mayor contenido de lisina en las tortillas en comparación con la del grano (Cuadro 3), se atribuye a la concentración de los componentes químicos debido principalmente a la pérdida de fibra (pericarpio), almidón y aceite durante el proceso de nixtamalización, como señalaron Coutiño et al. (2008). La concentración de lisina en las tortillas de los híbridos ACP y el testigo incre- mentó entre 16.1 y $32.8 \%$ en promedio, respectivamente, cuando los maíces se produjeron a densidad baja, y entre 20.8 y $29.9 \%$ cuando se cultivaron en densidad alta.

El contenido de triptófano en grano fue similar en ambas densidades, para los maíces ACP y el testigo (Cuadro 3). Entre genotipos los maíces ACP tuvieron $35 \%$ más triptófano que el testigo 'Promesa', diferencia menor a la informada por Vázquez et al. (2012) para los mismos híbridos. Durante el procesamiento a tortilla los maíces ACP y el testigo sembrados a densidad baja perdieron 30.4 y $12.9 \%$

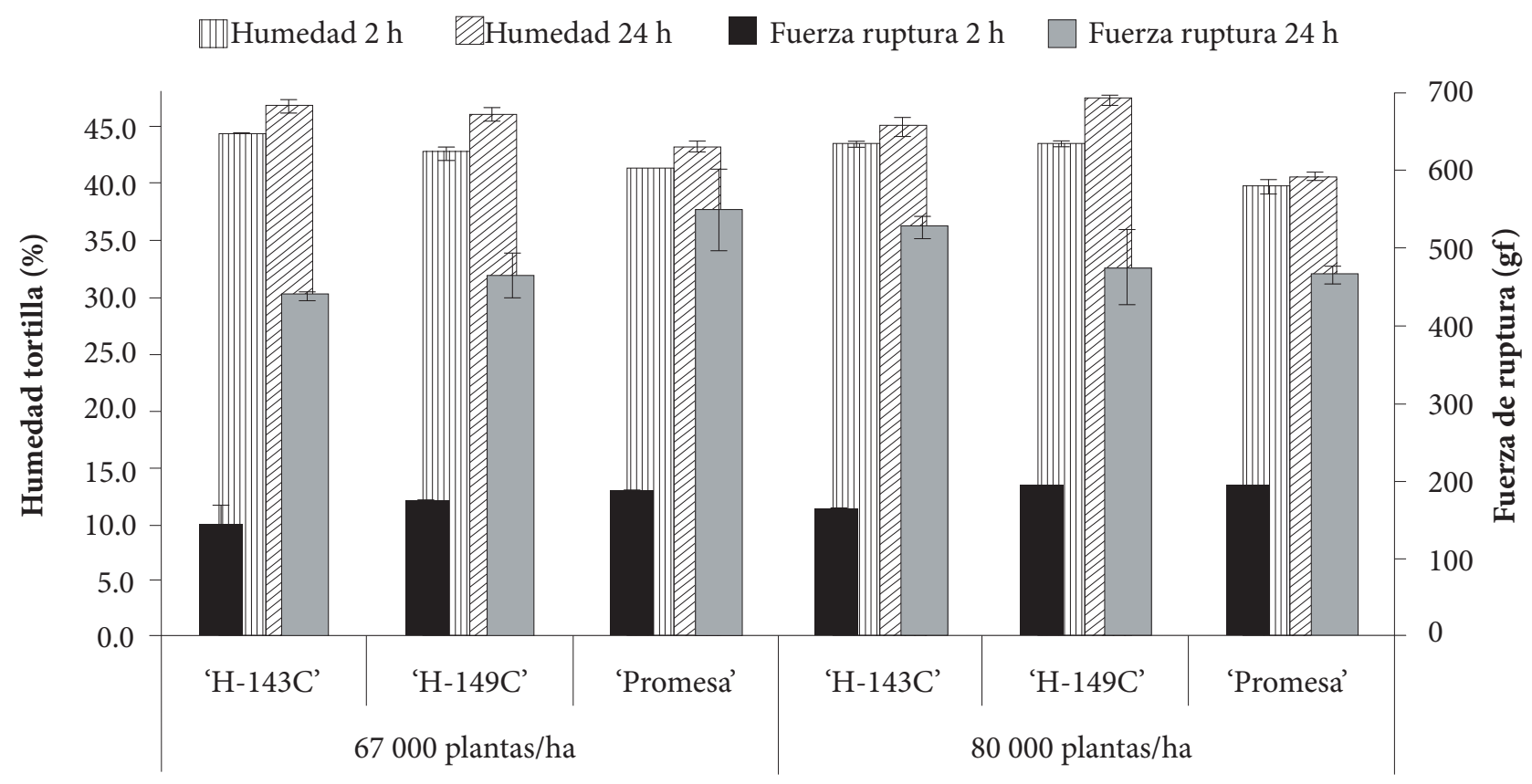

Densidad de población $\mathrm{x}$ híbrido

Figura 2. Humedad y textura de las tortillas de dos híbridos de alta calidad proteínica (ACP) y un testigo no ACP ('Promesa') producidos en Santa Lucía, Coatlinchán, Edo. de Méx., PV-2009, en dos densidades de población.

Cuadro 3. Comparación de medias de componentes químicos de dos híbridos de maíz de alta calidad proteínica (ACP) y un testigo no ACP ('Promesa'), sembrados en dos densidades de población. Santa Lucía, Coatlinchán, Edo. de Méx. PV-2009.

\begin{tabular}{|c|c|c|c|c|c|c|c|c|c|c|c|}
\hline \multirow{3}{*}{$\begin{array}{l}\text { Densidad } \\
\left(\text { Plantas ha }^{-1}\right)\end{array}$} & \multirow{3}{*}{ Híbrido } & \multirow{2}{*}{\multicolumn{2}{|c|}{$\begin{array}{c}\text { Proteína }{ }^{\dagger, \dagger \dagger} \\
(\%)\end{array}$}} & \multicolumn{2}{|c|}{ Lisina $^{\dagger \dagger}$} & \multicolumn{2}{|c|}{ Triptófano $^{\dagger \dagger}$} & \multicolumn{2}{|c|}{ Aceite } & \multirow{2}{*}{$\frac{\text { Almidón }^{\dagger \dagger}}{(\%)}$} & \multirow[t]{2}{*}{$\mathrm{AST}^{\dagger \dagger}$} \\
\hline & & & & & $(\mathrm{g} / 100 \mathrm{~g}$ & roteína) & & & & & \\
\hline & & Grano & Tortilla & Grano & Tortilla & Grano & Tortilla & Grano & Tortilla & Grano & Grano \\
\hline \multirow{3}{*}{67000} & 'H-143C' & $9.4 \mathrm{~d}$ & $9.1 \mathrm{c}$ & $3.87 \mathrm{a}$ & $4.30 \mathrm{a}$ & $0.87 \mathrm{a}$ & $0.65 \mathrm{a}$ & $3.6 \mathrm{c}$ & $2.9 \mathrm{~d}$ & $76.5 \mathrm{a}$ & $1.205 \mathrm{a}$ \\
\hline & 'H-149C' & $10.4 \mathrm{~b}$ & $9.1 \mathrm{c}$ & $3.38 \mathrm{~b}$ & $4.09 \mathrm{ab}$ & $0.76 \mathrm{a}$ & $0.49 \mathrm{~b}$ & $4.5 \mathrm{~b}$ & $3.6 \mathrm{bc}$ & $65.0 \mathrm{c}$ & $1.235 \mathrm{a}$ \\
\hline & 'Promesa' & $9.9 c$ & $9.4 \mathrm{~b}$ & $2.65 c$ & $3.52 \mathrm{bc}$ & $0.54 \mathrm{~b}$ & $0.47 \mathrm{bc}$ & $5.2 \mathrm{a}$ & $3.8 \mathrm{ab}$ & $70.0 \mathrm{bc}$ & $0.825 \mathrm{~b}$ \\
\hline \multirow{3}{*}{80000} & 'H-143C' & $9.5 \mathrm{~d}$ & $9.1 \mathrm{c}$ & $3.39 \mathrm{~b}$ & $4.37 \mathrm{a}$ & $0.83 \mathrm{a}$ & $0.68 \mathrm{a}$ & $3.8 \mathrm{c}$ & $3.0 \mathrm{~d}$ & $78.5 \mathrm{a}$ & $1.125 \mathrm{a}$ \\
\hline & 'H-149C' & $8.6 \mathrm{e}$ & $8.6 \mathrm{~d}$ & $3.94 \mathrm{a}$ & $4.44 \mathrm{a}$ & $0.78 \mathrm{a}$ & $0.62 \mathrm{a}$ & $3.9 c$ & $3.3 \mathrm{~cd}$ & $76.0 \mathrm{a}$ & $1.130 \mathrm{a}$ \\
\hline & 'Promesa' & $10.7 \mathrm{a}$ & $9.9 \mathrm{a}$ & $2.47 \mathrm{c}$ & $3.21 \mathrm{c}$ & $0.52 \mathrm{~b}$ & $0.39 c$ & $5.5 \mathrm{a}$ & $4.1 \mathrm{a}$ & $73.5 \mathrm{ab}$ & $0.880 \mathrm{~b}$ \\
\hline
\end{tabular}

Medias con letras iguales, en columnas, no son estadísticamente diferentes (Tukey, 0.05 ). ${ }^{\dagger} \mathrm{N}$ x $6.25 ;{ }^{\dagger \dagger}$ Muestra desgrasada y en base seca; ${ }^{99} \%$ en base seca; AST $=$ azúcares solubles totales (glucosa, fructosa y sacarosa). 
de triptófano, respectivamente. Al sembrarse en densidad alta las reducciones de triptófano fueron de 19.2 y $25.0 \%$ respectivamente. Pérdidas similares de estos aminoácidos fueron reportadas por Rojas et al. (2008) y por Vázquez et al. (2012).

En cuanto al contenido de aceite en grano solo el híbrido 'H-149C' mostró diferencias por efecto de la DP, pues a densidad baja presentó un contenido superior de aceite que a densidad alta (Cuadro 3). Las mayores pérdidas de aceite durante el proceso de elaboración de tortilla se presentaron en el testigo 'Promesa' ( $23.4 \%$, en promedio). En promedio las tortillas elaboradas a partir de los híbridos ACP tuvieron $3.2 \%$ de aceite, valor superior al reportado por Vázquez et al. (2012).

El contenido de almidón fluctuó en el intervalo de 65 a $78 \%$ entre los híbridos, y el mayor contenido de este componente se registró en los tres híbridos sembrados a la densidad alta de 80000 plantas ha $^{-1}$. Este comportamiento es contrario al informado por Sangoi (2000) quien afirmó que a mayores densidades de población se reduce el contenido de almidón debido a que se limita la capacidad de crecimiento del endospermo y se reduce el número de células y los gránulos de almidón formados. Luca y Tabara (2010) reportaron comportamientos similares a los encontrados en este estudio para aceite y almidón. Los maíces ACP tuvieron mayor contenido de azúcares totales solubles que el testigo 'Promesa' (Cuadro 3). No se encontraron diferencias significativas en el contenido de azúcares solubles por efecto de la interacción de los factores.

\section{CONCLUSIONES}

La calidad comercial y la composición química de los híbridos de maíz ACP, 'H-143C' y 'H-149C', se vio afectado por la densidad de plantación y su interacción con el genotipo. En el híbrido ' $\mathrm{H}-143 \mathrm{C}$ ' el efecto de ambos factores sobre las variables evaluadas fue mínimo, mientras que en el híbrido 'H-149C' una baja densidad de siembra (67 000 plantas ha ${ }^{-1}$ ) favoreció el desarrollo de granos más duros, más grandes, con mayor peso hectolítrico y mayor contenido de proteína, aceite y azúcares solubles totales, pero menos almidón que a mayor densidad (80 000 plantas ha1). Tanto el 'H-143C' como el testigo 'Promesa' cultivados en ambas densidades son aptos para su comercialización y procesamiento por las industrias de la masa y la tortilla. El 'H-149C' sembrado a densidades altas, debido a la suavidad del grano, solo es apto para el procesamiento doméstico. Estos dos híbridos ACP poseen una calidad de proteína mayor tanto en grano como en tortillas, por tener un mayor contenido de los aminoácidos esenciales lisina (4.2\%) y triptófano $(0.65 \%)$ que el híbrido testigo 'Promesa' (3.3 y $0.43 \%$, respectivamente), por lo que estos híbridos ACP se- rían benéficos para las personas que padecen desnutrición y consumen tortillas elaboradas con estos maíces.

\section{BIBLIOGRAFÍA}

AACC, American Association of Cereal Chemists (2000) Approved Methods of the AACC. 16th ed. AACC. The Association. St. Paul, MN. USA. 1200 p.

Ansari O, M Baga, R N Chibbar, N Sultana, N K Howes (2010) Analysis of starch swelling power in Australian breeding lines of hexaploid wheat (Triticum aestivum L.). Field Crops Res. 115:171178.

Antuna G O, S A Rodríguez H, G Arámbula V, A Palomo G, E Gutiérrez A, A Espinoza B, E F Navarro O, E Andrio E (2008) Calidad nixtamalera y tortillera en maíces criollos de México. Rev. Fitotec. Mex. 31(Especial 3):23-27.

Arámbula V G, J A Méndez A, J González H, E Gutiérrez A, E Moreno M (2004) Evaluación de una metodología para determinar características de textura de tortilla de maíz (Zea mays L.) Arch. Latinoam. Nutr. 54:216-222.

AOAC, Association of Official Analytical Chemists (1990) Official Methods of Analysis of the AOAC. 15th ed. AOAC, Washington DC, USA.

Carrera V J A y T Cervantes S (2006) Respuesta a densidad de población de cruzas de maíz tropical y subtropical adaptadas a Valles Altos. Rev. Fitotec. Mex. 29:331-338.

Coutiño E B, G Vázquez C, B Torres M, Y Salinas M (2008) Calidad de grano, tortillas y botanas de dos variedades de maíz de la raza comiteco. Rev. Fitotec. Mex. 31(Especial 3):9-14.

Dombrink K M A, C A Knutson (1997) A study of maize endosperm hardness in relation to amylose content and susceptibility to damage. Cereal Chem. 74:776-780.

Espinosa A, N Gómez, M Sierra, E Betanzos, F Caballero, B Coutiño, A Palafox, F Rodríguez, A García, O Cano (2003) Tecnología y producción de semillas de híbridos y variedades sobresalientes de maíz de calidad proteínica (QPM) en México. Agron. Mesoam. 14:223-228.

FAO, Food and Agriculture Organization of the United Nations (2013) Producción-Cultivos. Disponible en http://faostat3.fao.org/ home/index.html\#DOWNLOAD (Abril, 2013).

Galicia L, E Nurit, A Rosales, N Palacios R (2009) Laboratory Protocols 2009: Maize Nutrition Quality and Plant Tissue Analysis Laboratory. CIMMYT. México. $42 \mathrm{p}$.

Gupta H S, P K Agrawal, V Mahajan, G S Bisht, A Kumar, P Verma, A Srivastava, S Saha, R Babu, M C Pant, V P Mani (2009) Quality protein maize for nutritional security: rapid development of short duration hybrids through molecular marker assisted breeding. Curr. Sci. 96:230-237.

Herrera S R, J T Huber (1989) Influence of varying protein and starch degradabilities on performance of lactating cows. J. Dairy Sci. 72:1477-1483.

Luca S, V Tabara (2010) Effect of plant density on the main physical and chemical indicators of some maize hybrids cultivated in the Arad Plain. Res. J. Agric. Sci. 42:148-153.

Martínez B F, H E Martínez F, E Sanmartin M, F Sánchez S, Y K Chang, D B A, E Ríos (2001) Effect of the components of maize on the quality of masa and tortillas during the traditional nixtamalisation process. J. Sci. Food Agric. 81:1455-1462.

Mazón G M A, J S Escobedo G, E Herrera C, A Macías L, J Hernández P, G Vázquez C, P A Wesche E (2012) Maíz de alto contenido proteínico (Zea mays L.) en hogares rurales marginados del estado de Puebla. Est. Soc. 20:133-154.

Melesio C J L, R E Preciado O, A D Terrón I, M G Vázquez C, P Herrera M, C A Amaya G, S O Serna S (2008) Potencial productivo, propiedades físicas y valor nutrimental de híbridos de maíz de alta calidad proteínica. Agric. Téc. Méx. 34:225-233.

Mendoza E M, C Mosqueda V, J A Rangel L, A López B, S A Rodríguez H, L Latournerie M, E Moreno M (2006) Densidad de población y fertilización nitrogenada en la clorofila, materia seca y rendimiento de maíz normal y QPM. Agric. Téc. Méx. 32:89-99. 
Mendoza E M, N Moran V, E Andrio E, A López B, S A Rodríguez H, G Castañón N (2007) Efecto del nitrógeno y la densidad de población en el contenido de lisina de maíz en México. Agron. Mesoam. 18:177-183.

Norma Mexicana para Maíces Destinados al Proceso de Nixtamalización, NMX-FF034-2002-SCFI-Parte-1, NMX-034 (2002) Productos alimenticios no industrializados para consumo humano-cereales-Maíz blanco para proceso alcalino para tortillas de maíz y productos de maíz nixtamalizado- Especificaciones y métodos de prueba. Secretaria de Agricultura, Ganadería, Desarrollo Rural, Pesca y Alimentación; Dirección General de Normas. México, D.F. 18 p.

Palafox A, M Sierra M, A Espinosa C, F Rodríguez M, E N Becerra L (2008) Tolerancia a infestación por gorgojos (Sitophilus spp.) en genotipos de maíz comunes y de alta calidad proteínica. Agron. Mesoam. 19:39-46.

Polanco J A, T Flores M (2008) Bases para una Política de I\&D en Innovación de la Cadena de Valor del Maíz. Foro Consultivo Científico y Tecnológico. México, D. F. 244 p.

Rojas M I, E Gutiérrez, M E Cortés A, A Falcón, R Bressani, A Rojas, C Ibarra, J L Pons H, S H Guzmán M, A Cornejo V, M E Rodríguez (2008) Analysis of quality protein changes in nixtamlized QPM flours as a function of steeping time. Cereal Chem. 85:401-416.

Salinas M Y, G Vázquez C (2006) Metodologías de Análisis de la Calidad Nixtamalera-Tortillera en Maíz. Folleto Técnico No. 23. Instituto Nacional de Investigaciones Forestales, Agrícolas y Pecuarias. Campo Experimental Valle de México. Chapingo, Edo de México. México. 91 p.

Salinas M Y, J J López R, G B González F, G Vázquez C (2007) Compuestos fenólicos del grano de maíz y su relación con el oscurecimiento de masa y tortilla. Agrociencia 41:295-305.
Salinas M Y, N O Gómez M, J E Cervantes M, M Sierra M, A Palafox C, E Betanzos M, B Coutiño E (2010) Calidad nixtamalera y tortillera en maíces del trópico húmedo y sub-húmedo de México. Rev. Mex. Cien. Agríc. 1:509-523.

Sangoi L (2000) Understanding plan density effects on maize growth and development: an important issue to maximize grain yield. Ciencia Rural 31:159-168.

Scholes J D, P J Lee, P Horton, D H Lewis (1994) Invertase: understanding changes in the photosynthetic and carbohydrate metabolism of barley leaves infected with powdery mildew. New Phytol. 126:213-222.

Serna S S O, C A Amaya G, P Herrera M, J L Melesio C, R E Preciado O, A D Terron I, G Vazquez C (2008) Evaluation of the lime cooking and tortilla making properties of quality protein maize hybrids grown in Mexico. Plant Foods Hum. Nutr. 63:119-125.

Sierra M M, A Palafox C, G Vázquez C, F Rodríguez M, A Espinosa C (2010) Caracterización agronómica, calidad industrial y nutricional de maíz para el trópico mexicano. Agron. Mesoam. 21:21-29.

Vázquez C M G, D Escobedo M, A González C, A Turrent F, C Tut C (2005) Contenido de proteína, lisina y triptófano en maíces de calidad proteínica (ACP) con diferente manejo agronómico. Agric. Téc. Méx. 31:191-202.

Vázquez C M G, H Mejía A, C Tut C, N Gómez M (2012) Características de granos y tortillas de maíces de alta calidad proteínica desarrollados para los Valles Altos Centrales de México. Rev. Fitotec. Mex. 35:23-31.

Zepeda B R, A Carballo C, A Muñoz O, J A Mejía C, B Figueroa S, F V González C (2007) Fertilización nitrogenada y características físicas, estructurales y calidad de nixtamal-tortilla del grano de híbridos de maíz. Agric. Téc. Méx. 33:17-24. 